\title{
Tumor necrosis factor-like weak inducer of apoptosis regulates particle-induced inflammatory osteolysis via the p38 mitogen-activated protein kinase signaling pathway
}

\author{
ZHIQIANG ZHANG ${ }^{1,2}$, YONGCHAO FANG ${ }^{2}$, QIANG WANG ${ }^{2}$, YU SUN $^{2}$, CHUANZHI XIONG $^{2}$, \\ $\mathrm{LI} \mathrm{CAO}^{3}$, BEIYUE WANG ${ }^{1}$, NIRONG BAO ${ }^{1}$ and JIANNING ZHAO ${ }^{1}$
}

\begin{abstract}
${ }^{1}$ Department of Orthopedics, Jinling Hospital, School of Medicine, Southern Medical University, Nanjing, Jiangsu 210001; Departments of ${ }^{2}$ Orthopedics and ${ }^{3}$ Pathology, Subei People's Hospital of Jiangsu Province, Yangzhou, Jiangsu 225001, P.R. China
\end{abstract}

Received June 4, 2014; Accepted January 23, 2015

DOI: $10.3892 / \mathrm{mmr} .2015 .3529$

\begin{abstract}
Periprosthetic osteolysis is the predominant cause of artificial joint loosening. Previous studies have demonstrated that p38 mitogen-activated protein kinase (p38 MAPK) may be involved in periprosthetic osteolysis. Tumor necrosis factor (TNF)-like weak inducer of apoptosis (TWEAK) is a member of the TNF family and is a multifunctional cytokine, which regulates cellular proliferation, angiogenesis, inflammation and apoptosis via the p38 MAPK signaling pathway. The present study investigated the expression levels of TWEAK and p38 MAPK in periprosthetic interface membranes and in RAW264.7 monocyte/macrophage cells, which were treated with titanium (Ti) particle stimulation, with or without a p38 inhibitor (SB203580). This was performed to determine whether TWEAK was involved in the particle-induced inflammatory osteolysis via the p38 MAPK signaling pathway. The expression levels of TWEAK, p38 MAPK and phosphorylated (p-)p38 MAPK were evaluated in the periprosthetic interface membrane tissues and the RAW cells by reverse transcription-quantitative polymerase chain reaction and western blotting. The contents of interleukin- 6 and monocyte chemoattractant protein-1 in the supernatant were measured by ELISA. The results demonstrated that the expression levels of TWEAK and p-p38 MAPK increased in the periprosthetic interface membrane tissues and the RAW cells stimulated with Ti particles, suggesting that TWEAK was involved in
\end{abstract}

Correspondence to: Professor Jianning Zhao, Department of Orthopedics, Jinling Hospital, School of Medicine, Southern Medical University, 305 Zhongshan East Road, Nanjing, Jiangsu 210001, P.R. China

E-mail: zhaojianning.0207@163.com

Key words: tumor necrosis factor-like weak inducer of apoptosis, p38 mitogen-activated protein kinase, phosphorylated-p38 mitogenactivated protein kinase, prosthetic loosening, periprosthetic osteolysis, wear particles particle-induced inflammatory osteolysis via the p38 MAPK signaling pathway.

\section{Introduction}

Periprosthetic osteolysis is the predominant reason causing of loosening of artificial hip joints $(1,2)$. Wear particles induce monocytes-macrophages, fibroblasts and synovial cells in the tissue surrounding the joints to release inflammatory cytokines, including tumor necrosis factor (TNF)- $\alpha$, interleukin (IL)-6 and monocyte chemoattractant protein (MCP)-1 $(3,4)$. These inflammatory cytokines activate osteoclasts by osteoprotegerin/receptor activator of nuclear factor (NF)- $\mathrm{B}$ ligand and the $\mathrm{NF}-\kappa \mathrm{B}$ signaling pathway, resulting in loss of bone surrounding the prosthesis (5-7).

TNF-like weak inducer of apoptosis (TWEAK) is a novel member of the TNF superfamily of ligands (8). TWEAK is involved in a variety of biological processes by binding to its receptor, releasing pro-inflammatory cytokines, regulating the immune response and stimulating apoptosis, and is involved in tissue repair and regeneration (9). The pro-inflammatory effect of TWEAK has been observed in various cell types, including synovial cells, macrophages, monocytes and osteoclasts (10). p38 mitogen activated protein kinase (p38 MAPK) is one member of the MAPK family and is involved in regulating cellular responses to external stimuli by intracellular signal transmission (11). The activation of the p38 MAPK pathway activates NF- $\kappa \mathrm{B}$, which is involved in inflammation, by regulating the secretion of pro-inflammatory factors $(12,13)$.

However, few investigations have been performed on the effects of the 38 MAPK signaling pathway in particle-induced osteolysis, the specific function and mechanism of TWEAK in the pathogenesis of prosthetic aseptic loosening or its correlation with p38 MAPK. Previous studies have demonstrated that TWEAK exhibits pathogenic importance by activating the p38 MAPK signaling pathway in lupus nephritis and myositis diseases $(14,15)$.

The present study investigated the expression levels of TWEAK and p38 MAPK in periprosthetic interface 
membranes and in RAW264.7 monocyte/macrophage cells treated with titanium (Ti) particles to examine the effect of the TWEAK-p38 MAPK signaling pathway in particle-induced inflammatory osteolysis.

\section{Materials and methods}

Patients and samples. All experimental procedures were approved by the ethics committee of Southern Medical University and Jinling Hospital (Nanjing, China). Informed consent was obtained from all patients.

The synovium was obtained from patients who underwent surgical procedures for plica syndrome or discoid meniscus (2 males, 1 female; 12-41 years old), whereas osteoarthritic synovium was obtained from patients who underwent joint replacement for osteoarthritis (2 males, 1 female; $71-82$ years old). Periprosthetic interface membranes were obtained from patients undergoing surgical procedures for aseptic loosening ( 2 females, 1 male; 60-74 years old). The samples were obtained between May and June 2013 at Jinling Hospital.

Particle preparation. TiAl6V4 particles were obtained from the College of Materials Science and Engineering of Nanjing University of Technology (Nanjing, China). The TiAl6V4 particles had a mean diameter of $51.7 \mathrm{~nm}$. Stock solutions of TiPS were prepared at a concentration of $20 \mathrm{mg} / \mathrm{ml}$ in phosphate-buffered saline (PBS; Gibco Life Technologies, Carlsbad, CA, USA), and the particles were dispersed well by sonication for 10 min using Shumei KQ218 Ultrasonic Cleaning equipment (Kunshan Ultrasonic Instruments Co., Ltd., Jiangsu, China). The particles were autoclaved for $15 \mathrm{~min}$ at $121^{\circ} \mathrm{C}$ and 15 psi using a high-pressure steam sterilizer (AMSCO ${ }^{\circledR}$ Evolution $^{\circledR}$ Steam Sterilizer HC900; STERIS Corporation, Mentor, OH, USA).

Cell culture. The RAW264.7 mouse macrophage-like cell line, was obtained from China Center for Type Culture Collection (Shanghai, China) and cultured in RPMI-1640 medium (Gibco Life Technologies) supplemented with $10 \%$ fetal bovine serum (Sigma-Aldrich, St. Louis, MO, USA). The cell density was adjusted to a concentration of $2 \times 10^{6}$ cells $/ \mathrm{ml}$, and $1 \mathrm{ml}$ cell suspension was added into each well of 24-well culture plates (BD Biosciences, Franklin Lakes, NJ, USA) for culture in a $5 \% \mathrm{CO}_{2}$ incubator at $37^{\circ} \mathrm{C}$. The cells were divided into four groups: Group A, macrophage cells; Group B, macrophage cells + p38 MAPK inhibitor (10 $\mu \mathrm{mol} / 1$ SB203580; AdooQ Bioscience, Irvine, CA, USA); Group C, macrophage cells + Ti particles (0.1 mg/ml TiPs); Group D, macrophage cells + Ti particles + p38 MAPK inhibitor. The cells were centrifuged at $300 \mathrm{x} g$ for $10 \mathrm{~min}$ at $4^{\circ} \mathrm{C}$, and the cells and supernatant were harvested following $48 \mathrm{~h}$ culture in a $5 \% \mathrm{CO}_{2}$ incubator at $37^{\circ} \mathrm{C}$, then centrifuged at $300 \mathrm{x} \mathrm{g}$ for $10 \mathrm{~min}$ at $4^{\circ} \mathrm{C}$.

Histological evaluation. The harvested tissues were prepared for histological examination in order to evaluate the inflammatory response. The specimens were fixed in buffered $10 \%$ formalin (Sigma-Aldrich) in PBS for 4-6 $\mathrm{h}$ at room temperature, embedded in paraffin (Sigma-Aldrich) and then cut into $5 \mu \mathrm{m}$ tissue sections. The tissue sections were then stained with hematoxylin and eosin (Sigma-Aldrich). The expression levels of TWEAK and phosphorylated (p)-p38 MAPK were detected using a mouse anti-human TWEAK (cat. no. CABT-47857MH; Creative Biomart, Shirley, NY, USA), or rabbit polyclonal p-p38 MAPK monoclonal antibody (cat. no. sc-101758; 1:150 in PBS; Santa Cruz Biotechnology, Inc., Santa Cruz, CA, USA) and a horseradish peroxidase-conjugated anti-mouse secondary antibody (1:300 in PBS; Santa Cruz Biotechnology, Inc.) at room temperature for $4 \mathrm{~h}$, followed by color development using diaminobenzidine tetrahydrochloride (Santa Cruz Biotechnology, Inc.) (16). The cells were observed under a MX61A microscope (Olympus Corporation, Tokyo, Japan).

Gene expression of TWEAK and p38 MAPK. The total RNA was extracted from each specimen using TRIzol ${ }^{\circledR}$ reagent (Invitrogen Life Technologies, Carlsbad, CA, USA) and cDNA was obtained by reverse transcription (RT) using SuperScript First-Strand Synthesis system for PCR (Invitrogen Life Technologies), as described previously (17). RT-quantitative polymerase chain reaction (qPCR) was performed to evaluate the expression levels of TWEAK and p38 MAPK. The primers used are listed in Table I; the primer sequences were retrieved from PrimerBank (http://pga.mgh.harvard.edu/primerbank/) and the primers were prepared by MGH DNA Core facility (https://dnacore. mgh.harvard.edu/synthesis/index.shtml). The expression of GAPDH was quantified as an internal control. The PCR was conducted using SYBR Green PCR master mix (Applied Biosystems, Foster City, CA, USA), and an ABI Prism 7000 Sequence Detection system with ABI Prism 7000 software (Applied Biosystems). The PCR cycle conditions were: $95^{\circ} \mathrm{C}$ for $15 \mathrm{~min}, 35$ cycles of $94^{\circ} \mathrm{C}$ for $1 \mathrm{~min} ; 59^{\circ} \mathrm{C}$ for $1 \mathrm{~min}$, $72^{\circ} \mathrm{C}$ for $1 \mathrm{~min}$; and $72^{\circ} \mathrm{C}$ for $10 \mathrm{~min}$. The specificity of the amplification of the expected DNA fragments was confirmed using 2\% agarose gel electrophoresis and by analysis of the melting curves. An amplification reaction control with no RT enzyme was performed in order to assess the interference of potential genomic DNA in the RNA solution. The relative gene expression was calculated using the following equation: $2 \Delta \mathrm{CT} ;\left(\Delta \mathrm{CT}=\mathrm{CT}_{\mathrm{GAPDH}}-\mathrm{CT}_{\text {target }}\right)$.

Western blotting. Western blot analysis was used to determine the protein expression levels of TWEAK, p38 MAPK and p-p38 MAPK, as described previously (18). Periprosthetic interface membranes, osteoarthritic synovial tissues, normal synovial tissues and RAW264.7 cells were used for western blot analysis. The total cellular proteins were extracted using radioimmunoprecipitation lysis buffer (Sigma-Aldrich), containing $1 \mathrm{mM}$ phenylmethylsulfonyl fluoride (Beyotime Institute of Biotechnology, Lianyungang, China). The protein samples were separated by $8 \%$ SDS-PAGE and were transferred to polyvinylidene fluoride membranes (Shanghai Biyuntian Bio-Technology Co., Ltd., Shanghai, China). After blocking with 5\% blocking buffer [3\% bovine serum albumin in Tris-buffered saline with Tween-20 (Sigma-Aldrich, St. Louis, MO, USA)] the membranes were incubated with the following antibodies at $4^{\circ} \mathrm{C}$ overnight: Mouse monoclonal anti-p38 MAPK (cat. no. sc-271120; Santa Cruz Biotechnology, Inc.), mouse 
Table I. Gene primer sequences.

\begin{tabular}{lllr}
\hline Gene & \multicolumn{1}{c}{ Forward primer $\left(5^{\prime}-3^{\prime}\right)$} & \multicolumn{1}{c}{ Reverse primer (5'-3') } & Product (bp) \\
\hline TWEAK & CCTCGCAGAAGTGCACCTAA & ACACCATCCACCAGCAAGTC & 287 \\
p38MAPK & GCATAATGGCCGAGCTGTTG & TCATGGCTTGGCATCCTGTT & 130 \\
GAPDH & GCTGGTCATCAACGGGAAA & ACGCCAGTAGACTCCACGACA & 105 \\
\hline
\end{tabular}

TWEAK, tumor necrosis factor-like weak inducer of apoptosis; MAPK, mitogen-activated protein kinase.
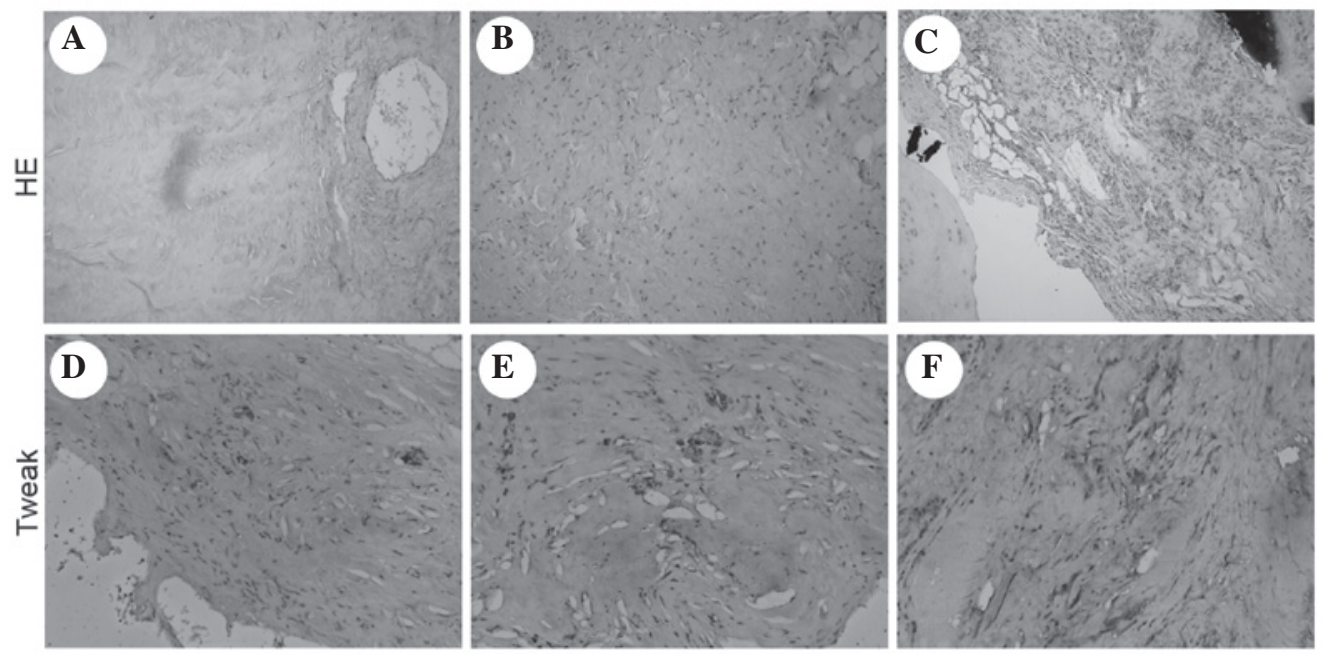

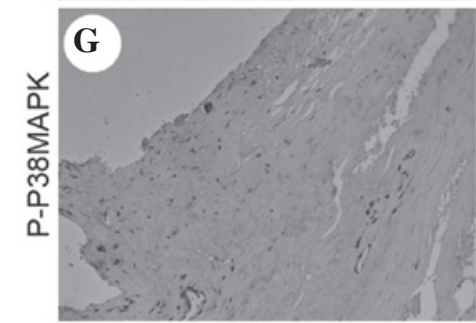

Normal synovium

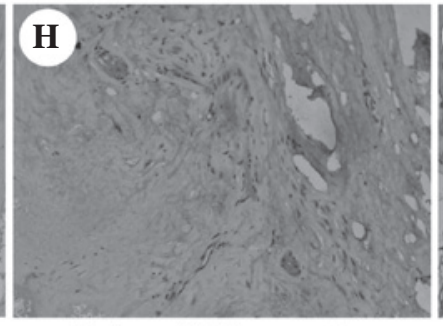

Osteoarthritis synovium

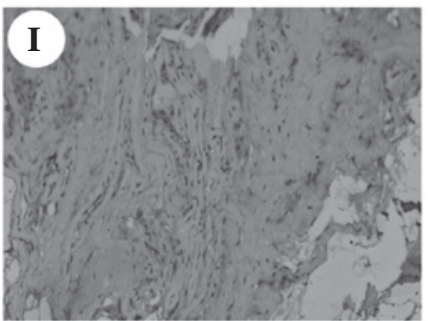

Interface membrane

Figure 1. Histological evaluation of (A) normal synovial tissue, (B) osteoarthritic synovial tissue and (C) interface membrane. The osteoarthritic synovial tissue contained a large number of fibrocytes and synovial cells compared with the normal synovial tissue. The osteoarthritic synovial tissue exhibited little inflammatory cell infiltration, while a marked inflammatory cell infiltration and hyperplasia was detected in synovial cells in interface membrane. These findings were further supported by the immunohistochemical analysis. TWEAK immunohistochemical staining in (D) normal synovial tissue, (E) osteoarthritic tissue and (F) interface membrane. P-p-38 MAPK immunohistochemical staining in (G) normal synovial tissue, (H) osteoarthritic synovial tissue and (I) interface membrane. A large quantity of inflammatory cells with positive staining of TWEAK and p-p38 MAPK were observed in the interface membrane, whereas fewer inflammatory cells were observed in the osteoarthritic synovial tissue. No positive staining of TWEAK and p-p38 MAPK was detected, indicating that no inflammatory response had occurred in the normal synovial tissue. HE, hematoxylin and eosin; TWEAK, tumor necrosis factor-like weak inducer of apoptosis; MAPK, mitogen-activated protein kinase; p-, phosphorylated.

monoclonal anti-p-p38 MAPK (cat. no. sc-7973; Santa Cruz Biotechnology, Inc.), goat polyclonal anti-TWEAK (cat. no. sc-12405; Santa Cruz Biotechnology, Inc.) and mouse monoclonal anti- $\beta$-actin (cat. no. sc-47778; Santa Cruz Biotechnology, Inc.), as described previously (19). The membranes were then incubated with alkaline phosphatase-conjugated secondary antibodies (cat. no. sc-2033; donkey anti-goat IgG; Santa Cruz Biotechnology, Inc.) at $37^{\circ} \mathrm{C}$ for $1 \mathrm{~h}$.. Visualization of the bound antibody was achieved using film (Kodak, Rochester, NY, USA) and 5-bromo-4-chloro-3-indolyl phosphate/nitroblue tetrazolium (Beyotime Institute of Biotechnology) at various time points ( $2 \mathrm{~min}, 10 \mathrm{~min}$ and $2 \mathrm{~h}$ ). The relative density of each protein band was determined using an Odyssey infrared imaging system (Li-Cor Biosciences, Lincoln, NE, USA).

Cytokine detection in the supernatant. The expression levels of IL-6 and MCP-1 in each group were quantified using ELISA kits (BD Biosciences Pharmingen, San Diego, CA, USA), according to the manufacturer's instructions. The experiments were performed in triplicate.

Statistical analysis. The data are expressed as the mean \pm standard deviation. The data from each group were analyzed by one-way analysis of variance, and statistical analyses were conducted using SPSS version 16.0 (SPSS Inc., Chicago, IL, 


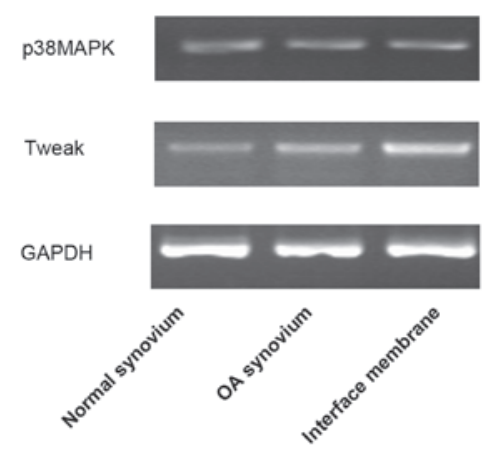

B

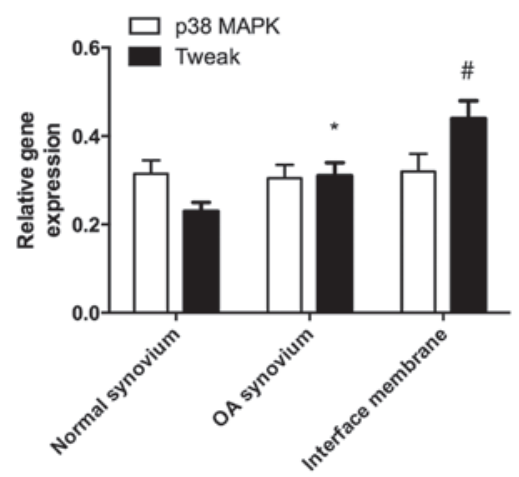

Figure 2. Gene expression of TWEAK and p38 MAPK. (A and B) mRNA expression levels of TWEAK in the interface membrane, osteoarthritic synovial tissue and normal synovial tissue were significantly different $(\mathrm{P}<0.05)$. No significant difference in the mRNA expression of $\mathrm{p} 38 \mathrm{MAPK}$ was observed between the three groups $(\mathrm{P}>0.05)$. ${ }^{*} \mathrm{P}<0.05$, vs. normal synovium; ${ }^{*} \mathrm{P}<0.05$, vs. OA synovium. MAPK, mitogen-activated protein kinase; TWEAK, tumor necrosis factor-like inducer of apoptosis; OA, osteoarthritic synovial tissue.

A

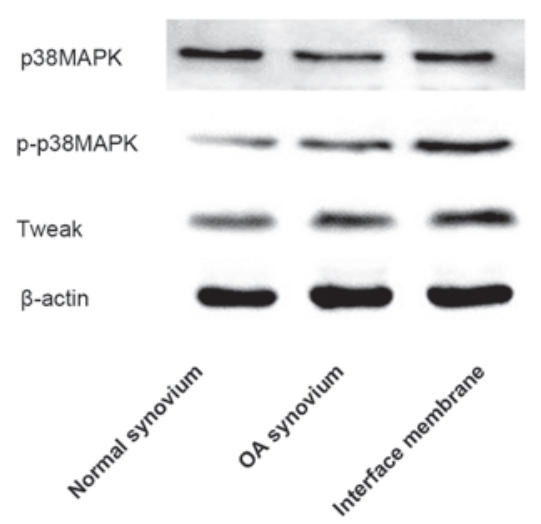

B

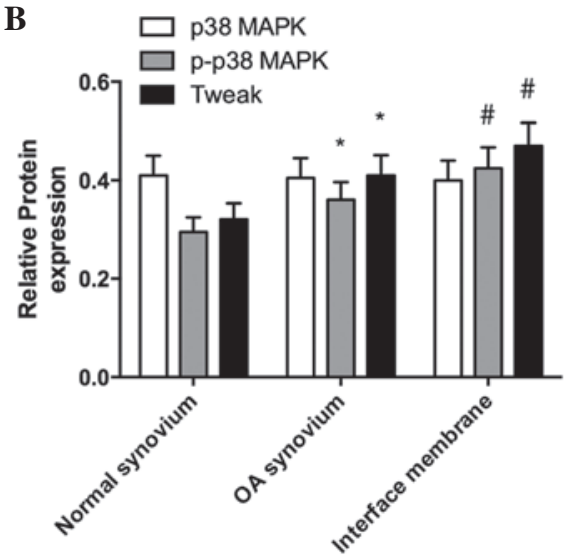

Figure 3. Western blot analysis to determine the expression levels of TWEAK, p-p38 MAPK and p38 MAPK in the different tissues. (A) Representative western blot analysis and (B) quantification of band densities demonstrated that there was a significant difference in the protein expression levels of TWEAK and p-p38 MAPK between the interface membrane, osteoarthritic synovial tissue and normal synovial tissue. The protein expression levels of p38 MAPK in the three groups demonstrated no significant difference. ${ }^{*} \mathrm{P}<0.05$, vs. normal synovium; ${ }^{*} \mathrm{P}<0.05$, vs. OA synovium. MAPK, mitogen-activated protein kinase; TWEAK, tumor necrosis factor-like inducer of apoptosis; OA, osteoarthritic synovial tissue; p-, phosphorylated.

USA). $\mathrm{P}<0.05$ was considered to indicate a statistical significant difference.

\section{Results}

Histological evaluation. The osteoarthritic synovial tissue contained more fibrocytes and synovial cells compared with the normal synovial tissue (Fig. 1) and little inflammatory cell infiltration. Marked inflammatory cell infiltration and hyperplasia of synovial cells were observed in the interface membrane.

These findings were further supported by the results of the immunohistochemical analyses. Increased levels of inflammatory cells with positive staining of TWEAK and p-p38 MAPK were observed in the interface membrane, whereas fewer inflammatory cells were observed in the osteoarthritic synovial tissue. In addition, no positive staining of TWEAK or p-p38 MAPK was detected, indicating that no inflammatory response had occurred in the normal synovial tissue.

Gene expression of TWEAK and 38 MAPK. The mRNA expression of TWEAK in the interface membrane was markedly higher in the normal tissue compared with the osteoarthritic synovial tissue, whereas the mRNA expression of TWEAK in the osteoarthritic synovial tissue was markedly increased compared with the normal synovial tissue. No significant difference in the mRNA expression of p38 MAPK was observed between the three groups (Fig. 2).

Protein expression levels of TWEAK, p-p38 MAPK and p38 MAPK in the tissue sections. The expression levels of TWEAK and p-p38 MAPK were increased in the interface membrane compared with the osteoarthritic synovial tissue, whereas the mRNA expression levels of TWEAK and p38 MAPK in the osteoarthritic synovial tissue were higher compared with the normal synovial tissue. No significant difference in the protein expression of p38 MAPK was observed between the three groups (Fig. 3)

Effect of the SB203580 p38 MAPK inhibitor on the protein expression levels of TWEAK, p38 MAPK and p-p38 MAPK in RAW246.7 cells. As shown in Fig. 4, the protein expression of TWEAK in group $C$ was higher compared with the other 
A

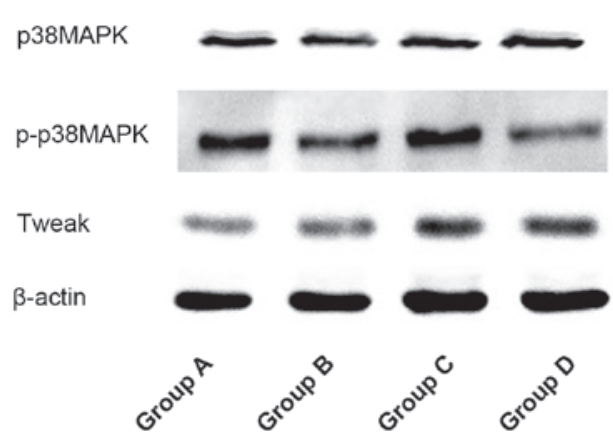

B

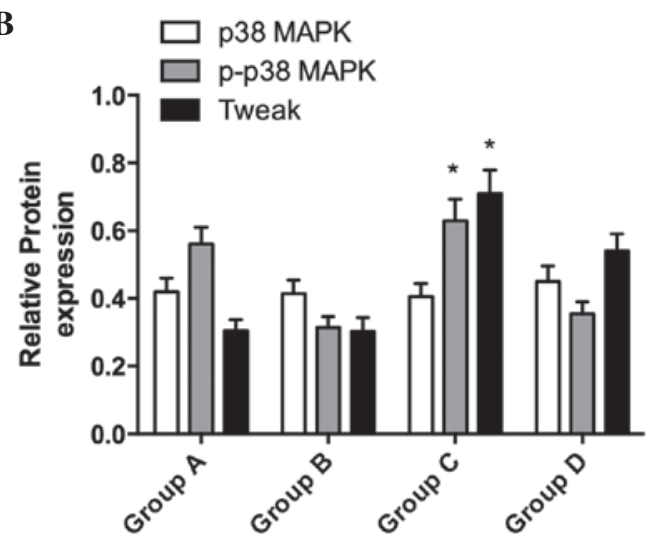

Figure 4. Protein expression levels of TWEAK, p38 MAPK and p-p38 MAPK in untreated RAW246.7 cells (Group A) and those treated with p38 MAPK inhibitor (Group B); Ti particles (Group C); or Ti particles + p38 MAPK inhibitor (Group D). (A) Representative western blot analysis and (B) quantification of band densities demonstrated that the protein expression of TWEAK in group $\mathrm{C}$ was higher compared with the other groups $(\mathrm{P}<0.05)$ and that the protein expression of TWEAK in group D was higher compared with groups A and B $(\mathrm{P}<0.05)$. No significant difference between groups $\mathrm{A}$ and $\mathrm{B}$ were observed $(\mathrm{P}>0.05)$. The protein expression of $\mathrm{p}$-p38 MAPK in group $\mathrm{C}$ was higher compared with the other groups $(\mathrm{P}<0.05)$ and the protein expression of $\mathrm{p}$-p $38 \mathrm{MAPK}$ in group $\mathrm{A}$ was higher compared with groups B and $\mathrm{D}(\mathrm{P}<0.05)$. No significant difference in the protein expression of p-p38 MAPK was observed between groups B and $\mathrm{D}(\mathrm{P}>0.05)$, and no significant difference was observed in the protein expression levels of p38 MAPK in any of the groups $(\mathrm{P}>0.05)$. "P $<<0.05$, vs. group $\mathrm{A}$, group B and group D. MAPK, mitogen-activated protein kinase; TWEAK, tumor necrosis factor-like inducer of apoptosis; Ti, titanium; p-, phosphorylated.

groups $(\mathrm{P}<0.05)$ and the protein expression of TWEAK in group D was higher compared with those in groups A and B $(\mathrm{P}<0.05)$, which indicated that the Ti particles stimulated the upregulation of the expression of TWEAK. No significant difference was observed between groups $\mathrm{A}$ and $\mathrm{B}(\mathrm{P}>0.05)$.

The protein expression of p-p38 MAPK in group $C$ was higher compared with the other groups $(\mathrm{P}<0.05)$ and the protein expression of p-p38 MAPK in group A was higher compared with groups $B$ and $D(P<0.05)$. No significant difference in the protein expression of p-p38 MAPK was observed between groups $\mathrm{B}$ and $\mathrm{D}(\mathrm{P}>0.05)$. No differences were observed in the protein expression of $\mathrm{p} 38 \mathrm{MAPK}$ in the four groups $(\mathrm{P}>0.05)$.

Levels of IL-6 and MCP-1 in the supernatant of cultured RAW246.7 cells. The levels of IL-6 and MCP-1 in group C were significantly higher compared with the other groups $(\mathrm{P}<0.05$; Fig. 5). No significant difference was observed between groups $\mathrm{A}$ and $\mathrm{B}(\mathrm{P}>0.05)$.

\section{Discussion}

Aseptic loosening caused by periprosthetic osteolysis is the most serious complication following total hip arthroplasty (20). It is reported that debris particle-induced inflammation is the main cause of osteolysis, therefore, limiting the inflammatory response of the membrane, induced by debris particles, has become an area of interest (21). Several studies have demonstrated that macrophages and foreign body giant cells surrounding the prosthetic wear debris are the central effector cells and regulatory cells of inflammation, which secret various cytokines, including TNF- $\alpha$, IL-1, IL- 6 and MCP-1 $(22,23)$. Ingham and Fisher reported that the level of osteolysis is significantly decreased following the neutralization of TNF- $\alpha$, which indicates that TNF- $\alpha$ is involved in the osteolytic process, either directly or indirectly (22).

The MAPK cascade is an important intracellular signal transduction system. p38 MAPK is a classical pathway of

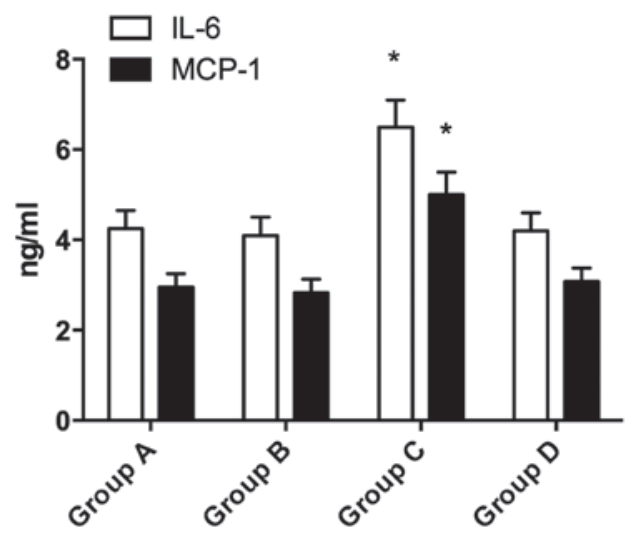

Figure 5. Levels of IL-6 and MCP-1 in the supernatants of cultured in untreated RAW246.7 cells (Group A) and those treated with p38 MAPK inhibitor (Group B); Ti particles (Group C); or Ti particles + p38 MAPK inhibitor (Group D). The levels of IL-6 and MCP-1 in group C were higher compared with the other groups $(\mathrm{P}<0.05)$. No significant difference was observed between groups $\mathrm{A}$ and $\mathrm{B}(\mathrm{P}>0.05)$. ${ }^{*} \mathrm{P}<0.05$, vs. group A, group $\mathrm{B}$ and group $\mathrm{D}$. IL, interleukin; MCP, monocyte chemoattractant protein; Ti. titanium.

the MAPK family, which is involved in the inflammatory process (24). The p38 MAPK pathway is activated by a variety of physical and chemical factors, inflammatory factors, stress stimuli and Gram-positive bacterial cell wall components, which causes effector cells to secrete a variety of inflammatory cytokines involved in the inflammatory response following injury (14). It has been reported the p38 MAPK pathway is important in periprosthetic osteolysis, which may be regulated by TWEAK $(14,25)$.

TWEAK is a novel member of the TNF ligand family and is a type II membrane protein, which is expressed in a variety of human tissues, including cartilage, skeletal muscle, macrophages and peripheral blood lymphocytes (26). TWEAK is important in immune system-mediated tissue damage, and the physiology and pathology of the disease, which includes the secretion of growth factors, cell proliferation and apoptosis (27). 
In the present study, the interface membrane tissue, normal synovial tissue and osteoarthritic synovial tissue were compared. The results of histological staining demonstrated that cell proliferation was significantly increased and accompanied by focal inflammatory cell infiltration in the interface membrane group compared with the other two groups. The existence of inflammatory cells in the surrounding tissue of the prosthetic loosening demonstrated that inflammation was important in particle-induced osteolysis.

Several studies using an animal model of particle-induced osteolysis have suggested that p38 MAPK is important in inflammatory osteolysis $(10,28)$. The present study performed quantitative detection of the p-p38 MAPK protein in the three tissue groups and demonstrated that the protein expression of p-p38 MAPK in the interface membrane tissue was higher compared with the normal synovial tissue and osteoarthritic synovial tissue, which confirmed that p38 MAPK was important in prosthetic loosening. Additionally, the findings demonstrated that the protein expression of p-p38 MAPK was higher in the osteoarthritic synovial tissue compared with the normal synovial tissue, which indicated that p-p38 MAPK exerted pro-inflammatory effects in acute and chronic inflammation.

In the present study, the detection of TWEAK by RT-qPCR and western blotting revealed similar results. The gene expression levels of TWEAK in the three tissue groups were consistent with the protein expression levels in the three groups, which suggested that the gene and protein expression levels in the interface membrane tissue were higher compared with the normal synovial tissue and osteoarthritic synovial tissue. The expression of TWEAK in the arthritis synovial group was higher compared with the normal synovial group, which was consistent with the results of van Kuijk et al (29), which confirmed the inflammatory effect of TWEAK. In addition, the data also suggested that there was a positive correlation between the expression of TWEAK and the expression of p-p38 MAPK in the three groups. According to the above results, it was hypothesized that TWEAK was involved in the pathological process of particle-induced inflammatory osteolysis in the loosening of prostheses.

It has been suggested that there is a clear positive correlation between TWEAK and p38 MAPK, and that TWEAK is involved in the secretion of proinflammatory cytokines and chemokines, cell proliferation and other biological effects $(14,30)$. Therefore, the present study hypothesized that the increased expression of TWEAK may promote inflammatory osteolysis and aimed to examine the molecular mechanisms of TWEAK in inflammatory osteolysis and determine whether this protein is important for the prevention of aseptic loosening of artificial joints

Previous studies have demonstrated that macrophages and foreign body giant cells are stimulated by prosthetic wear particles, and that these cells are the central effector cells and regulatory cells in inflammation $(21,22,31)$. Therefore, macrophages (RAW246.7) in the interface membrane were selected as study models, and Ti particles were used to stimulate the cells, which were simultaneously treated with the SB203580 p38 MAPK inhibitor to detect the secretion of IL- 6 and MCP-1. The results demonstrated that the protein levels of TWEAK and p-p38 MAPK and the IL-6 and MCP-1 content of the supernatant in group $\mathrm{C}$ was higher compared with groups $\mathrm{A}$ and $\mathrm{B}(\mathrm{P}<0.05)$, which indicated that macrophages under particle stimulation produced a significant inflammatory response. The expression levels of p-p38 MAPK, IL-6 and MCP-1 in group D were lower compared with group C $(\mathrm{P}<0.05)$, which indicated that the macrophage inflammatory response was suppressed by the inhibition of p-p38 MAPK.

No significant differences in the expression of TWEAK or in the concentrations of IL-6 and MCP-1 in the supernatant were observed between groups A and B, however, the expression of p-p38 MAPK was suppressed significantly. The expression of TWEAK in group D was higher compared with groups A and B, whereas no significant difference were observed in the secretion of IL- 6 or MCP-1 in the supernatant between groups A, B and D. These findings indicated that Ti particles stimulated macrophages and promoted the expression levels of TWEAK and p-p38 MAPK. TWEAK exerted its inflammatory effects dependently and independently of the p38 MAPK signaling pathway, and was only stimulated by the Ti particles. The expression levels of IL-6 and MCP-1 were significantly dependent on the p38 MAPK signaling pathway.

In conclusion, the present study hypothesized that wear particles surrounding the prosthesis stimulate macrophages to secrete TWEAK and simultaneously activate the p38 MAPK pathway. TWEAK activated the p38 MAPK pathway by binding its receptor and induced the secretion of inflammatory cytokines, including IL-6 and MCP-1, which caused periprosthetic inflammatory osteolysis. In addition, MCP-1 promoted the aggregation of the monocyte-macrophage cells, further exacerbating the periprosthetic inflammatory osteolytic response. This indicated that TWEAK/p38 MAPK, IL-6 and MCP-1 signaling are important in particle-induced inflammatory osteolysis. Therefore, further investigation into the mechanism underlying p38 MAPK signaling pathways in periprosthetic inflammatory osteolysis may provide a novel mechanism to inhibit osteolysis and is important for the prevention and treatment of aseptic loosening of artificial joints.

\section{Acknowledgements}

The present study was supported by the Jiangsu Clinical Science and Technology project (grant no. BL2012002).

\section{References}

1. Moro T, Kyomoto M, Ishihara K, et al: Grafting of poly(2-methacryloyloxyethyl phosphorylcholine) on polyethylene liner in artificial hip joints reduces production of wear particles. J Mech Behav Biomed Mater 31: 100-106, 2014.

2. Fujii J, Yasunaga Y, Yamasaki A and Ochi M: Wear debris stimulates bone-resorbing factor expression in the fibroblasts and osteoblasts. Hip Int, 2011.

3. Antonios JK, Yao Z, Li C, Rao AJ and Goodman SB: Macrophage polarization in response to wear particles in vitro. Cell Mol Immunol 10: 471-482, 2013.

4. Nich C, Takakubo Y, Pajarinen J, et al: Macrophages-Key cells in the response to wear debris from joint replacements. J Biomed Mater Res A 101: 3033-3045, 2013.

5. Shimamura M, Nakagami H, Osako MK, et al: OPG/RANKL/RANK axis is a critical inflammatory signaling system in ischemic brain in mice. Proc Natl Acad Sci USA 111: 8191-8196, 2014. 
6. Ma B, Zhang Q, Wu D, et al: Strontium fructose 1,6-diphosphate prevents bone loss in a rat model of postmenopausal osteoporosis via the OPG/RANKL/RANK pathway. Acta Pharmacol Sin 33 : 479-489, 2012

7. Zhang X, Paun A, Claudio E, Wang H and Siebenlist U: The tumor promoter and $\mathrm{NF}-\kappa \mathrm{B}$ modulator $\mathrm{Bcl}-3$ regulates splenic B cell development. J Immunol 191: 5984-5992, 2013.

8. Dwyer BJ, Olynyk JK, Ramm GA and Tirnitz-Parker JE: TWEAK and LT $\beta$ signaling during chronic liver disease. Front Immunol 5: 39, 2014.

9. Martin P,MoraI,Cortes A, et al: Relevant role of cGMP-dependent protein kinase (PKG) in the progression of fibrosis induced by TNF-like weak inducer of apoptosis (TWEAK). Am J Physio Renal Physiol 307: F75-F85, 2014.

10. Winkles JA: The TWEAK-Fn14 cytokine-receptor axis: discovery, biology and therapeutic targeting. Nat Rev Drug Discov 7: 411-425, 2008.

11. Yang Q, Xu Y, Feng G, et al: p38 MAPK signal pathway involved in anti-inflammatory effect of Chaihu-Shugan-San and Shen-ling-bai-zhu-San on hepatocyte in non-alcoholic steatohepatitis rats. Afr J Tradit Complement Altern Med 11: 213-221, 2014

12. Moreira V, Lomonte B, Vinolo MA, Curi R, Gutierrez JM and Teixeira C: An Asp49 phospholipase A2 from snake venom induces cyclooxygenase-2 expression and prostaglandin E2 production via activation of NF-kappa B, p38MAPK, and PKC in Macrophages. Mediators Inflamm 2014: 2014.

13. Wang $X$ and Liu Y: Regulation of innate immune response by MAP kinase phosphatase-1. Cell Signal 19: 1372-1382, 2007.

14. Zhi-Chun L, Qiao-Ling Z, Zhi-Qin L, Xiao-Zhao L, Xiao-xia Z and Rong T: Tumor necrosis factor-like weak inducer of apoptosis (TWEAK) mediates p38 mitogen-activated protein kinase activation and signal transduction in peripheral blood mononuclear cells from patients with lupus nephritis. Inflammation 35 935-943, 2012

15. Li H, Mittal OK, Paul PK, et al: Tumor necrosis factor-related weak inducer of apoptosis augments matrix metalloproteinase 9 (MMP-9) production in skeletal muscle through the activation of nuclear factor-kappaB-inducing kinase and p38 mitogen-activated protein kinase: A potential role of MMP-9 in myopathy. J Biol Chem 284: 4439-4450, 2009

16. Wisniacki N, Amaravadi L, Galluppi GR, et al: Safety, tolerability, pharmacokinetics, and pharmacodynamics of anti-TWEAK monoclonal antibody in patients with rheumatoid arthritis. Clin Ther 35: 1137-1149, 2013.

17. Xue K, Qi L, Zhou G and Liu K: A two-step method of constructing mature cartilage using bone marrow-derived mesenchymal stem cells. Cells Tissues Organs 197: 484-495, 2013
18. Schultz DC, Bazel S, Wright LM, et al: Western blotting and enzymatic activity analysis of cathepsin D in breast tissue and sera of patients with breast cancer and benign breast disease and of normal controls. Cancer Res 54: 48-54, 1994.

19. Xiao W, Chen X and He M: Inhibition of the Jagged/Notch pathway inhibits retinoblastoma cell proliferation via suppressing the PI3K/Akt, Src, p38MAPK and Wnt//catenin signaling pathways. Mol Med Rep 10: 453-458, 2014

20. Purdue PE, Koulouvaris P, Potter HG, Nestor BJ and Sculco TP: The cellular and molecular biology of periprosthetic osteolysis. Clin Orthop Relat Res 454: 251-261, 2007.

21. Gallo J, Goodman SB, Konttinen YT and Raska M: Particle disease: biologic mechanisms of periprosthetic osteolysis in total hip arthroplasty. Innate Immun 19: 213-224, 2013.

22. Ingham $E$ and Fisher J: The role of macrophages in osteolysis of total joint replacement. Biomaterials 26: 1271-1286, 2005.

23. Pajarinen J, Kouri VP, Jamsen E, Li TF, Mandelin J and Konttinen YT: The response of macrophages to titanium particles is determined by macrophage polarization. Acta Biomater 9: 9229-9240, 2013

24. Goodman SB, Ma T, Spanogle J, et al: Effects of a p38 MAP kinase inhibitor on bone ingrowth and tissue differentiation in rabbit chambers. J Biomed Mater Res A 81: 310-316, 2007.

25. Tsirigotis M, Baldwin RM, Tang MY, Lorimer IA and Gray DA: Activation of p38MAPK contributes to expanded polyglutamine-induced cytotoxicity. PloS One 3: e2130, 2008.

26. Desplat-Jégo S, Feuillet L, Creidy R, et al: TWEAK is expressed at the cell surface of monocytes during multiple sclerosis. J Leukoc Biol 85: 132-135, 2009.

27. Mao X, Pan X, Peng X, Cheng T and Zhang X: Inhibition of titanium particle-induced inflammation by the proteasome inhibitor bortezomib in murine macrophage-like RAW264.7 cells. Inflammation 35: 1411-1418, 2012.

28. Goodman SB, Gibon E, Pajarinen J, et al: Novel biological strategies for treatment of wear particle-induced periprosthetic osteolysis of orthopaedic implants for joint replacement. J R Soc Interface 11: 20130962, 2014

29. van Kuijk AW, Wijbrandts CA, Vinkenoog M, Zheng TS, Reedquist KA and Tak PP: TWEAK and its receptor Fn14 in the synovium of patients with rheumatoid arthritis compared to psoriatic arthritis and its response to tumour necrosis factor blockade. Ann Rheum Dis 69: 301-304, 2010.

30. Perper SJ, Browning B, Burkly LC, et al: TWEAK is a novel arthritogenic mediator. J Immunol 177: 2610-2620, 2006.

31. Neale SD and Athanasou NA: Cytokine receptor profile of arthroplasty macrophages, foreign body giant cells and mature osteoclasts. Acta Orthop Scand 70: 452-458, 1999. 